\title{
OTX2 Signals from the Choroid Plexus to Regulate Adult Neurogenesis
}

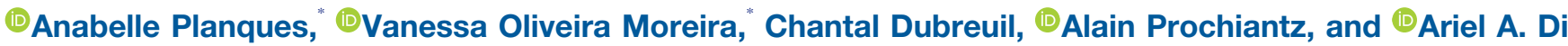 \\ Nardo
}

https://doi.org/10.1523/ENEURO.0262-18.2019

Centre for Interdisciplinary Research in Biology, Collège de France, CNRS UMR 7241, INSERM U1050, PSL Research University, 75006 Paris, France

\begin{abstract}
Proliferation and migration during adult neurogenesis are regulated by a microenvironment of signaling molecules originating from local vasculature, from CSF produced by the choroid plexus, and from local supporting cells including astrocytes. Here, we focus on the function of OTX2 homeoprotein transcription factor in the mouse adult ventricular-subventricular zone (V-SVZ), which generates olfactory bulb neurons. We find that OTX2 secreted by choroid plexus is transferred to the supporting cells of the V-SVZ and rostral migratory stream. Deletion of Otx2 in choroid plexus affects neuroblast migration and reduces the number of olfactory bulb newborn neurons. Adult neurogenesis was also decreased by expressing secreted single-chain antibodies to sequester OTX2 in the CSF, demonstrating the importance of non-cell-autonomous OTX2. We show that OTX2 activity modifies extracellular matrix components and signaling molecules produced by supporting astrocytes. Thus, we reveal a multilevel and non-cell-autonomous role of a homeoprotein and reinforce the choroid plexus and astrocytes as key niche compartments affecting adult neurogenesis.
\end{abstract}

Key words: astrocyte; CSF; extracellular matrix; homeoprotein

\section{Significance Statement}

CSF, local vasculature and non-neurogenic astrocytes are niche compartments that provide a microenvironment for regulating adult mouse neurogenesis. We show that OTX2 homeoprotein secreted by choroid plexus into the CSF is transferred into non-neurogenic astrocytes of the ventricular-subventricular zone and a rostral migratory stream where it regulates extracellular matrix and signaling factors. This non-cellautonomous activity impacts the number of newborn neurons that integrate the olfactory bulb. Thus, we reveal a multilevel role for OTX2 and reinforce the choroid plexus as a key niche compartment affecting adult neurogenesis.

\section{Introduction}

Neurogenesis in the adult mouse brain provides continuous replacement of interneurons in olfactory bulbs (OBs) and is important for olfaction-based learning (Lledo and Valley, 2016). Neural stem cells (NSCs), located in the adult ventricular-subventricular zone (V-SVZ) lining the lateral cerebral ventricles, give rise to progenitor cells (the first transient amplifying cells that generate migrating neu- roblasts) that migrate though the rostral migratory stream (RMS) to reach the OB where they differentiate as interneurons and integrate into local circuitry. This process is regulated not only by factors intrinsic to the NSCs and progenitors but also by their microenvironment consisting of factors from local vasculature, neuronal circuits, CSF, and local supporting cells (Lehtinen et al., 2013; SilvaVargas et al., 2013; Bjornsson et al., 2015). It has been 
shown that NSCs contact vascular endothelial cells and project into the ventricular wall where their cilia contact CSF, which participates in regulating proliferation and migration through mechanical influence and CSF-borne factors (Sawamoto et al., 2006; Mirzadeh et al., 2008; Shen et al., 2008; Petrik et al., 2018).

The CSF is produced within brain ventricles by the choroid plexuses (ChPs), which express many secreted factors found to influence neurogenesis, including growth factors, morphogens, and guidance clues (Falcão et al., 2012; Kokovay et al., 2012; Silva-Vargas et al., 2016). Examples of CSF-borne factors include IGF2 (insulin like growth factor 2), which promotes adult NSC renewal (Ziegler et al., 2015); IGF1 and BMP5 (bone morphogenetic protein 5), which promote NSC colonies (SilvaVargas et al., 2016); NT-3 (neurotrophin-3), which promotes quiescence (Delgado et al., 2014); and SLIT2 (slit guidance ligand 2), a chemorepulsive factor that participates in neuroblast migration (Nguyen-Ba-Charvet et al., 2004; Sawamoto et al., 2006). Neuroblasts and NSCs also contact local non-neurogenic astrocytes (Lois et al., 1996; Shen et al., 2008), which secrete factors that help to establish and maintain the microenvironment and can influence neurogenesis (for review, see Gengatharan et al., 2016). Such factors include DLK1 (delta-like noncanonical notch ligand 1), which induces NSC selfrenewal (Ferrón et al., 2011), and thrombospondin 4 (THBS4), which guides neuroblast migration (Girard et al., 2014).

Homeoproteins are key regulators of neurogenesis both during embryogenesis and in the adult (Curto et al., 2014; Prochiantz and Di Nardo, 2015). This class of transcription factors has the property to act both cell autonomously and non-cell autonomously after secretion and internalization in target cells. The Otx2 homeoprotein is a regulator of embryonic development and embryonic neurogenesis (Yang et al., 2014; Hoch et al., 2015; Acampora et al., 2016), but its role in adult neurogenesis has not been investigated. OTX2 is expressed by ChP, secreted in CSF, and transfers in brain parenchyma to regulate cortical plasticity in postnatal mice (Spatazza et al., 2013; Lee et al., 2017). We hypothesized that OTX2 in the CSF might also accumulate in adult neurogenic niches and impact neurogenesis. Indeed, we find that V-SVZ neurogenesis is regulated by OTX2 expression and secretion by the ChP, and find that OTX2 transfers into supporting astrocytes to control the expression of extracellular factors that impact neuroblast migration.

This work was supported by Région lle-de-France via le DIM (les domaines d'intérêt majeur) Cerveau et Pensée, by European Research Council Advanced Grant HOMEOSIGN \#339379, and by Korean Global Reasearch Laboratory Program \#2009-00424.

*A. Planques and V.O.M. contributed equally to this work.

Correspondence should be addressed to Ariel A. Di Nardo at Ariel.dinardo@college-de-france.fr.

https://doi.org/10.1523/ENEURO.0262-18.2019

Copyright (C) 2019 Planques et al.

This is an open-access article distributed under the terms of the Creative Commons Attribution 4.0 International license, which permits unrestricted use, distribution and reproduction in any medium provided that the original work is properly attributed.

\section{Materials and Methods}

\section{Animals}

S129 mice were purchased from Charles River Laboratories. The Otx2 $2^{\text {lox/lox }}$ (Otx2-lox) mice (S129 background) were donated by Dr. T. Lamonerie, Institut de Biologie Valrose, Nice, France (Fossat et al., 2006), and Otx2 $2^{+/ G F P}$ mice were donated by $\mathrm{Dr}$. A. Simeone, Institute of Genetics and Biophysics, Napoli, Italy (Acampora et al., 2009). The single-chain antibody (scFv) knock-in mouse model (scFv-Otx2 mice, C57BL/6J background) were previously described (Bernard et al., 2016). Adult mice (3 months old) of either sex were used in all experiments, with littermates distributed equally between control and treated groups while maintaining sex balance. All animal procedures, including housing, were conducted in accordance with the guidelines of the European Economic Community (2010/63/UE) and the French National Committee (2013/ 118). This research (project \#00704.02) was approved by Ethics Committee 59 of the French Ministry for Research and Higher Education. Mice were conventionally raised (12 h light/dark cycle) in cages with red tunnels, nesting cotton, and food and water available ad libitum.

\section{Mouse surgery}

For surgical procedures, animals were anesthetized with xylazine (2\% Rompun, Bayer; $5 \mathrm{mg} / \mathrm{kg}$ ) and ketamine (Imalgene 500, Merial; $80 \mathrm{mg} / \mathrm{kg}$ ). Cre-Tat recombinant protein $(8-20 \mu \mathrm{g} / \mathrm{ml}$ in $400 \mathrm{mM} \mathrm{NaCl}, 15 \%$ DMSO) or vehicle was injected as previously described (Spatazza et al., 2013). Ten days after Cre-Tat injection, mice were injected intraperitoneally with $150 \mathrm{mg} / \mathrm{kg}$ BrdU (B9285, Sigma-Aldrich; at $10 \mathrm{mg} / \mathrm{ml}$ diluted in $\mathrm{NaCl} 0.9 \%$ ). For OB integration experiments, mice were injected with BrdU twice daily for $5 \mathrm{~d}$, and then housed for 3 weeks before perfusion with PBS. For proliferation experiments, mice were injected with one pulse of BrdU and then perfused with PBS $2 \mathrm{~h}$ later. Brains were cut in half along the anterior-posterior axis: the anterior half was fixed for $48 \mathrm{~h}$ at $4{ }^{\circ} \mathrm{C}$ in $4 \%$ formaldehyde-PBS, immersed overnight in $20 \%$ sucrose PBS, and processed for cryostat sectioning; the posterior half was used to recover fourth ventricle $\mathrm{ChP}$ for quantitative PCR analysis of Otx2 expression.

\section{Astrocyte cell culture}

Primary astrocytes were prepared from cortex of newborn mouse [postnatal day 0 (P0) to P4] of either sex and cultured in $10 \mathrm{ml}$ of DMEM high glucose, $10 \%$ serum, $1 \times$ antimitotic/antibiotic (Même et al., 2006). After 7 d, astrocytes were passaged into 6-well plates at 400,000 cells/ $\mathrm{ml}$, incubated for $14 \mathrm{~d}$, and then treated with $1 \%$ Ara-C (in DMEM high glucose, $1 \%$ B27, $1 \times$ antimitotic/antibiotic) for $24 \mathrm{~h}$ before treatment with $100 \mathrm{ng} / \mathrm{ml}$ OTX2 protein. After $24 \mathrm{~h}$, treated astrocytes were processed for quantitative PCR analysis.

\section{Immunohistochemistry and histology}

Frozen brains were sectioned ( 25 or $40 \mu \mathrm{m}$ ) by cryostat and stored at $-20^{\circ} \mathrm{C}$. For all staining experiments, slides were thawed for $15 \mathrm{~min}$ at room temperature, hydrated in PBS, and incubated for antigen retrieval in a steamer for 
15 min with $10 \mathrm{~mm}$ citrate buffer, $\mathrm{pH}$ 6. After cooling, sections were washed with PBS and incubated in blocking buffer $[0.5 \%$ Triton-X, $10 \%$ normal donkey serum (Abcam) in PBS] for $30 \mathrm{~min}$. Primary antibodies were incubated overnight at room temperature [1/100 rat antiBrdU OBT0030, AbD Serotec (RRID:AB_609568); 1/400 rabbit anti-caspase \#9661, Cell Signaling Technology (RRID:AB_2341188); 1/250 rabbit anti-OTX2 ab92326 Abcam (RRID:AB_10562130); 1/1000 guinea pig anti-DCX (doublecortin) AB2253, Millipore (RRID:AB_1586992); 1/200 mouse anti-GFAP G6171, Sigma-Aldrich (RRID: AB 1840893); $1 / 1000$ chicken anti-Vimentin AB5733, Millipore (RRID:AB_11212377); 1/1000 chicken anti-GFP ab13970, Abcam (RRID:AB_300798)]. After PBS washes, secondary antibodies (1/2000 donkey anti-lgG Alexa Fluor, Invitrogen; RRID:AB_142924, RRID:AB_2535794, RRID:AB 141637, RRID:AB 141607) were incubated for 2 $h$ at room temperature. For DCX labeling, sections were washed with PBS, incubated for $2 \mathrm{~h}$ at room temperature with secondary antibody (1/500 biotinylated goat antiguinea pig ab6907, Abcam; RRID:AB_954847), washed again with PBS, and incubated for $2 \mathrm{~h}$ with conjugated streptavidin (1/2000 Alexa Fluor Streptavidin, Invitrogen; RRID:AB_2336066). Sections were washed in PBS, dried, and mounted in DAPI Fluoromount-G (Southern Biotech). TUNEL was performed with the in situ cell death detection kit (11684817910 Roche) according to manufacturer instructions. In situ hybridization was performed as previously described (Spatazza et al., 2013).

\section{Imaging and quantification}

Imaging was performed with an SP5 inverted confocal microscope (Leica), a Nikon 90i upright wide-field microscope, or a Spinning-disk confocal microscope with constant parameters. For BrdU analysis, one $40 \mu \mathrm{m}$ coronal section every $200 \mu \mathrm{m}$ was quantified. BrdU counting in the granule cell layer (GCL) and glomerular layer (GL) from the same mice was performed on six sections per animal from bregma +4.0 to its anterior end using an in-house ImageJ macro that automatically detects cells within an $\mathrm{ROI}$. The cell number was normalized by the ROI area for each image; the mean for each animal was reported. This method was validated in initial experiments where counting by hand in parallel gave similar results. BrdU counting in the V-SVZ was performed by hand on $8 \times 40 \mu \mathrm{m}$ coronal sections every $200 \mu \mathrm{m}$ per animal, from bregma +1.34 to bregma $-0.58 \mathrm{~mm}$, which represents the anterior V-SVZ in which proliferation is more sustained (Fiorelli et al., 2015). Cell numbers were normalized by ventricular wall length.

\section{Quantitative PCR}

Total RNA from fourth ventricle ChP was extracted with the RNeasy Lipid Tissue Mini Kit (Qiagen) with DNA removal. RNA and protein were extracted for extracellular matrix (ECM) composition analysis using the AllPrep DNA RNA/Protein Mini Kit (Qiagen). RNA was processed with the QuantiTect Reverse Transcription Kit (Qiagen). cDNA was diluted 1:10 with RNase-free water for quantitative PCR samples, which were analyzed in triplicate with a LightCycler 480 II (Roche) and SYBR Green I Master mix.
After $T_{m}$ profile validation, gene expression was determined by the $2^{-\Delta \Delta C t}$ method with hypoxanthine guanine phosphoribosyl transferase as the control gene. For expression analysis, the mean expression of vehicleinjected mice or control-treated cells was used for comparison.

\section{Microdissection}

For biochemical analysis, mice were killed by cervical elongation. Brain was placed in cold PBS, dorsal side facing up, and two equidistant longitudinal incisions in each OB were made to divide them into three parts while keeping them attached to the rest of the brain. The V-SVZ microdissection was performed as previously described (Mirzadeh et al., 2010). Afterward, the hemispheres were placed once again dorsal side facing up and the two sagittal cuts starting from the $O B$ incisions were pursued along the entire length of the brain. The central piece of tissue was laid on its side, and RMS was excised (see Fig. $2 D$ ). All tissue samples were stored at $-80^{\circ} \mathrm{C}$.

\section{Immunoprecipitation and Western blot}

Microdissections were triturated in $100 \mu$ l of lysis buffer [150 mM NaCl, 1\% Triton X-100, 100 mm Tris, pH 7.4, 10 mM EDTA, $1 \times$ protease/phosphatase inhibitor (Roche)] per $10 \mathrm{mg}$ of tissue and incubated $10 \mathrm{~min}$ on ice. Supernatant was recovered in low-bind tubes after centrifugation for $10 \mathrm{~min}$ at $16,000 \times \mathrm{g}$. Antibody was added [2.5 $\mu \mathrm{g}$ of anti-OTX2 (ab21990, Abcam) or anti-lgG (ab27478, Abcam)] and incubated overnight at $4{ }^{\circ} \mathrm{C}$ with rotation. Protein A Sepharose beads (GE Healthcare) were washed twice and resuspended 1:1 with lysis buffer. Beads $(30 \mu \mathrm{l})$ were added to each sample and incubated $3 \mathrm{~h}$ at $4^{\circ} \mathrm{C}$. Beads were recovered by centrifugation for $1 \mathrm{~min}$ at $16,000 \times g$ and washed five times with $1 \mathrm{ml}$ of cold wash buffer (Lysis buffer with $500 \mathrm{~mm} \mathrm{NaCl}$ ). Proteins were eluted from the beads with $30 \mu \mathrm{l}$ of $2 \times$ Laemmli buffer, heated for $5 \mathrm{~min}$ at $95^{\circ} \mathrm{C}$, and stored at $-20^{\circ} \mathrm{C}$.

Samples were separated on NuPage $4-12 \%$ Bis-Tris precast gels (Invitrogen) with $1 \times$ MES buffer and antioxidant (Invitrogen) then processed for Western blotting for OTX2 detection. Membranes were blocked in 5\% nonfat milk, TBS, $0.2 \%$ Tween, incubated with anti-OTX2 (inhouse mouse monoclonal) overnight at $4^{\circ} \mathrm{C}$ then incubated with HRP-conjugated anti-mouse (GeneTex) for $2 \mathrm{~h}$ at room temperature. A chemiluminescence reaction was performed with ECL Femto (Thermo Fisher Scientific). These immunoprecipitation experiments were performed three times, with similar results each time.

\section{Statistical analysis}

Prism 8 software (GraphPad Software) was used for all statistical analyses. Sample size was determined after preliminary experiments. Quantification was normalized by the mean value of vehicle-injected mice samples. Normal data distribution was assessed by the D'Agostino and Pearson omnibus normality test. If a normal test was not passed, the Mann-Whitney test was applied; otherwise, an unpaired $t$ test with Welch's correction was used (note $t$ test in figure legends). See figure legends for relevant statistical tests, and see Table 1 for results. 
Table 1: Statistical analyses

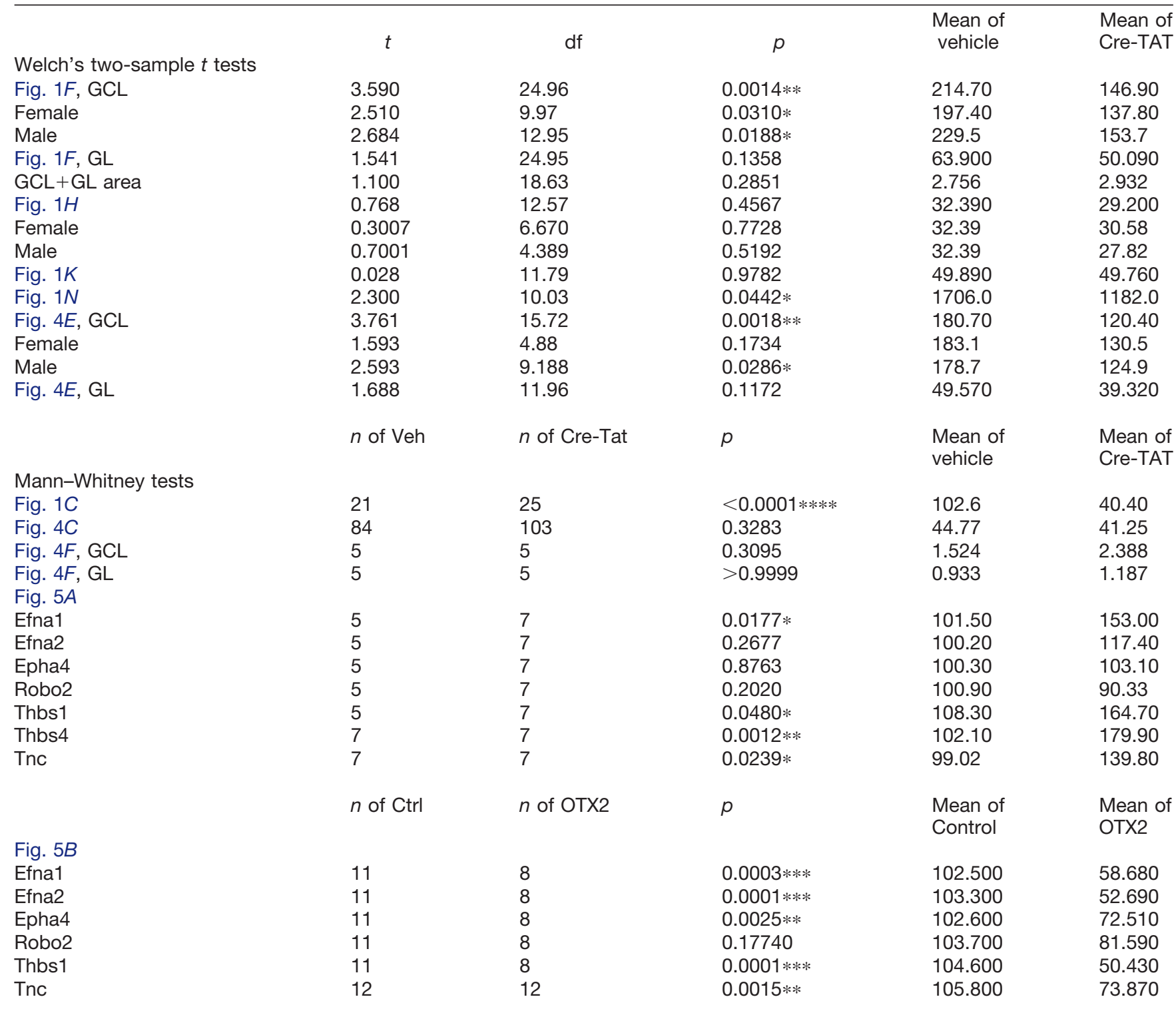

$* p<0.05, * * p<0.01, * * * p<0.001, * * * * p<0.0001$.

\section{Results}

\section{Otx2 knockdown in choroid plexus reduces newborn neurons in the olfactory bulb}

Intracerebroventricular injections of vectorized Cre recombinase (Cre-Tat) leads to specific recombination of Otx2 in lateral ventricle ChP of Otx2-lox mice (Spatazza et al., 2013). With this paradigm, we find that Cre-Tat intracerebroventricular injection leads to a strong decrease in OTX2 protein in lateral ventricle ChP compared with vehicle-injected mice (Fig. $1 A$ ) and also a decrease in Otx2 mRNA expression in both lateral and fourth ventricle ChP (Fig. 1B). While the decrease is not necessarily proportional between both ChPs for a given mouse, when a decrease of $>30 \%$ was observed in one ChP, a decrease of at least $30 \%$ was observed in the other ChP (Fig. 1B). Therefore, the fourth ventricle ChP quantitative PCR analysis is a valid method to assess Otx2 expression levels in lateral $\mathrm{ChP}$ so that anterior tissues can be used for histologic quantification. Otx2-lox mice with $<30 \%$ Otx2 expression knockdown in the fourth ventricle after Cre-Tat intracerebroventricular injection where excluded from subsequent analysis. For the experiments described below, Cre-Tat injections in lateral cerebral ventricles lead to $\sim 60 \%$ decrease in Otx2 mRNA expression on average in the fourth ventricle ChP (Fig. 1C).

To assess whether Otx2 knockdown in ChP alters V-SVZ neurogenesis, we used BrdU treatments to measure either newborn neuron integration in $\mathrm{OB}(3$ weeks postinjection; Fig. $1 D-F)$ or cell proliferation in V-SVZ $(2 \mathrm{~h}$ postinjection; Fig. 1/-K) after Cre-Tat or vehicle intracerebroventricular injections. We found a significant decrease in the density of GCL newborn neurons but no change in the density of GL newborn neurons after Otx2 knockdown (Fig. 1F). This change in density was not due to gross 
A
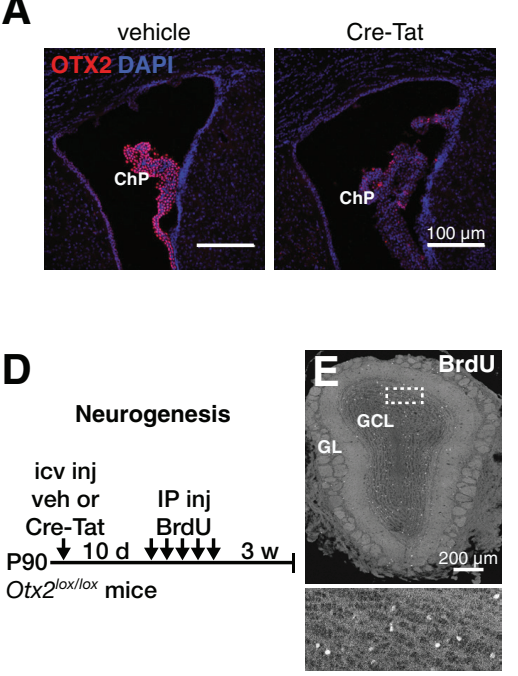

B

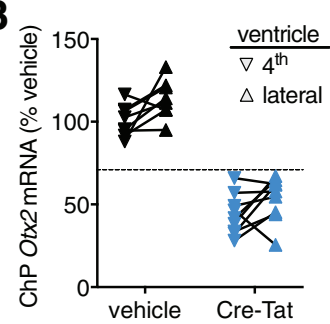

F

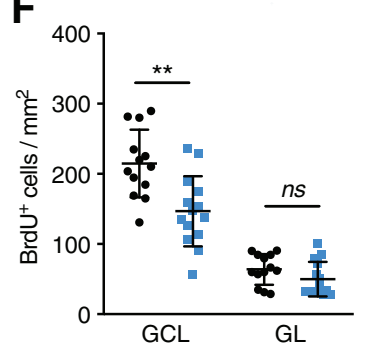

C

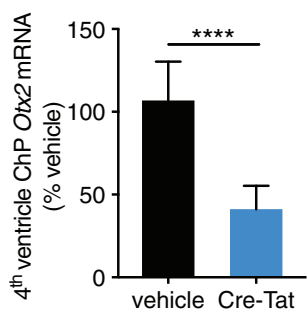

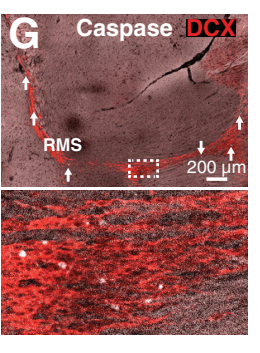

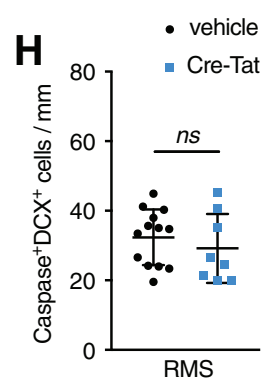

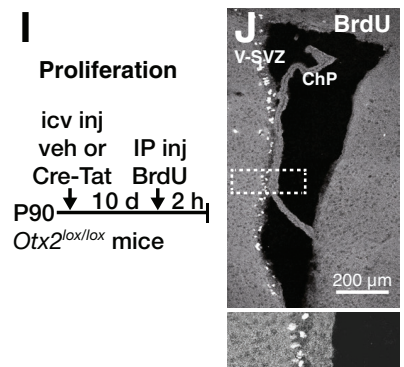
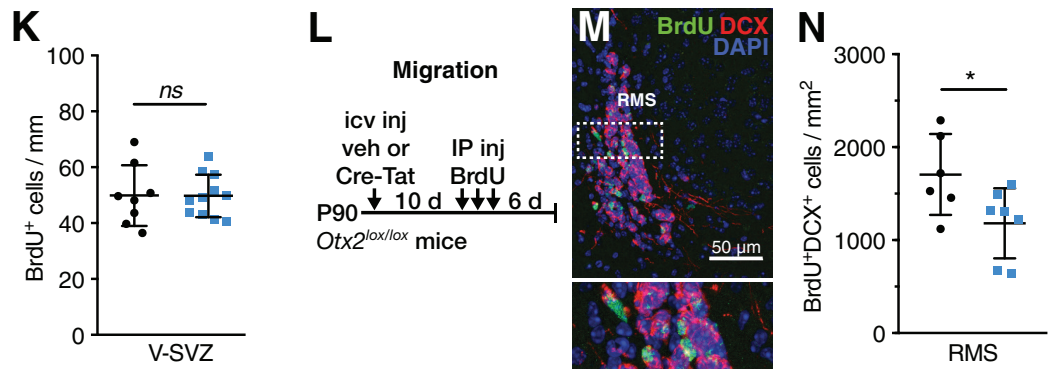

Figure 1. OTX2 knockdown in the choroid plexus reduces newborn neurons in the olfactory bulb. $\boldsymbol{A}-\boldsymbol{C}$, Conditional Otx2 knockdown with Otx2 $2^{10 x / l o x}$ mice. A, Staining for OTX2 in lateral ventricle choroid plexus $35 \mathrm{~d}$ after intracerebroventricular injection of vehicle or Cre-Tat. B, Comparison of Otx2 expression levels between fourth and lateral ventricles from the same brain (linked data points represent individual mouse) after vehicle or Cre-Tat intracerebroventricular injection. Dotted line represents $30 \%$ decrease in Otx2 expression. C, Quantitative PCR analysis of Otx2 expression in fourth ventricle (****p $<0.0001$, Mann-Whitney test, 5 experiments). $\boldsymbol{D}$, Schematic of adult (3 months old) neurogenesis study paradigm. $\boldsymbol{E}$, Staining for BrdU in a coronal section of olfactory bulb 3 weeks after BrdU injections. $\boldsymbol{F}$, Quantification of BrdU-positive cells in GCL and GL of the olfactory bulb ( $* * p<0.01, \mathrm{~ns}=p>0.05, t$ test, 3 experiments). G, Staining for caspase and DCX in a sagittal section of the RMS. Arrows indicate caspase-labeled neuroblasts throughout the entire RMS. $\boldsymbol{H}$, Quantification of caspase/DCX colabeled cells in RMS (ns $=p>0.05, t$ test, 3 experiments). $\boldsymbol{I}$, Schematic of adult V-SVZ proliferation study paradigm. J, Staining for BrdU in a coronal section of the V-SVZ $2 \mathrm{~h}$ after BrdU injection. K, Quantification of BrdU-positive cells in V-SVZ ( $\mathrm{ns}=p>0.05, t$ test, 2 experiments). $\boldsymbol{L}$, Schematic of adult migration study paradigm. $\boldsymbol{M}$, Staining for BrdU and DCX in a coronal section of the RMS. $\boldsymbol{N}$, Quantification of BrdU/DCX colabeled cells in RMS (*p $<0.05, t$ test, 2 experiments). IP, Intraperitoneal; icv, intracerebroventricular; inj, injection; veh, vehicle. Error bars represent SD.

structural changes as no significant change in $\mathrm{OB}$ area was observed (Table 1, GCL +GL area) and was not dependent on sex (Table 1). Analysis of caspase-expressing cells revealed no change in cell death in the RMS of Cre-Tat-injected mice (Fig. 1G,H), suggesting that OTX2 does not affect neuroblast survival. However, Otx2 knockdown in ChP does not alter V-SVZ cell proliferation (Fig. $1 K$ ), suggesting that the observed decrease in newborn neurons is due to effects on neuroblast migration. To test this hypothesis, we used a migration paradigm in which we evaluated the density of BrdU/DCX colabeled cells in the RMS $6 \mathrm{~d}$ after final BrdU injection (Fig. $1 L-N)$. We observed a significant decrease in Cre-Tatinjected animals, suggesting that fewer neuroblasts are leaving the V-SVZ and/or that they are migrating slower (Fig. 1N).

\section{Non-cell-autonomous Otx2 in V-SVZ and RMS}

Given that OTX2 is secreted from ChP into the CSF and accumulates in brain parenchyma (Spatazza et al., 2013), we hypothesized that OTX2 transfers from ChP to the V-SVZ niche. The Otx2 locus is silent in V-SVZ and RMS, as shown by the absence of GFP expression in Ot $x 2^{+/ G F P}$ mice (Fig. $2 A, B$ ) and by Otx2 in situ hybridization (Fig. 2C). However, OTX2 protein is immunoprecipitated from lysates of V-SVZ and RMS microdissections (Fig. 2D) but not from striatum lysates (microdissection beneath $V$ SVZ); Iysates of superior colliculus, in which Otx2 locus is active, were used as positive controls (Fig. 2E). Immunostaining showed the selective presence of OTX2 in a subset of cells within the V-SVZ and RMS (Fig. 3), suggesting that it could be targeting either neural stem cells or progenitor cells directly and/or other cells 

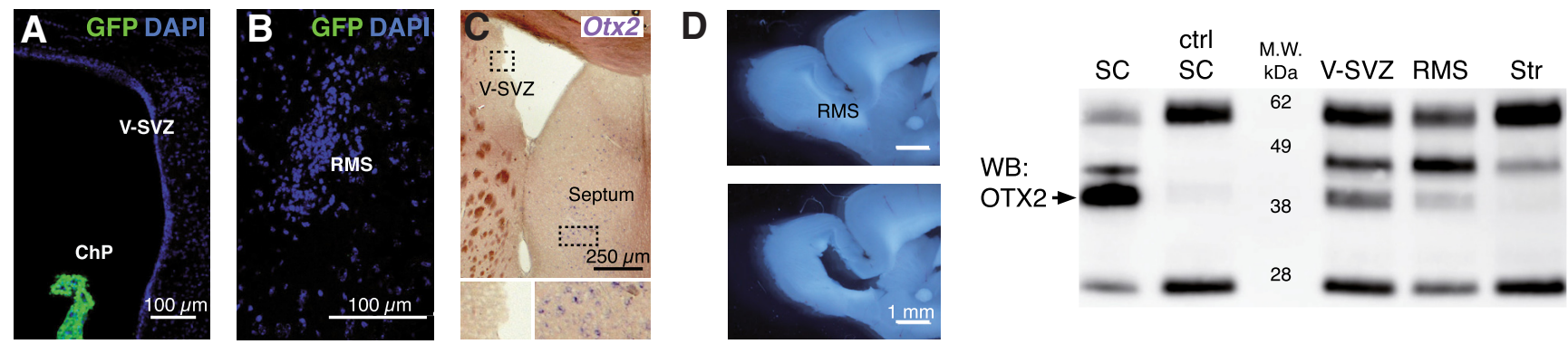

Figure 2. OTX2 in RMS and V-SVZ is non-cell-autonomous. $\boldsymbol{A}-\boldsymbol{C}$, Absence of Otx2 expression in V-SVZ (A, $\boldsymbol{C})$ and RMS (B), as shown by GFP staining in coronal sections of Otx2 $2^{+/ G F P}$ mice $(\boldsymbol{A}, \boldsymbol{B})$ and by in situ hybridization for Otx2 mRNA in coronal sections of wild-type mice $(\boldsymbol{C})$. Note the expression of GFP in choroid plexus $(\boldsymbol{A})$ and of Otx2 in septum (C). $\boldsymbol{D}$, Representative sagittal section of RMS microdissection. $\boldsymbol{E}$, Western blot (WB) analysis of OTX2 immunoprecipitation from lysates of various brain regions (ctrl, lgG control). Bands include OTX2 ( $<40 \mathrm{kDa})$, IgG $(25$ and $55 \mathrm{kDa})$, and unknown ( $45 \mathrm{kDa})$. SC, Superior colliculus; Str, striatum; M.W., molecular weight.

within the niche. OTX2 is not internalized in neuroblasts $\left(\mathrm{DCX}^{+}\right.$cells) in V-SVZ but accumulates in a small number of GFAP-labeled cells and many ependymal cells (Fig. $3 A, B)$. In and around the RMS, OTX2 is again absent in neuroblasts yet is in many GFAP-labeled cells (Fig. 3C,D).

OTX2 protein in regions of neuroblast proliferation and migration suggests that reduced newborn neurons in $\mathrm{OB}$ after Otx2 ChP knockdown in Otx2-lox mice (Fig. 1F) could be due in part to OTX2 non-cell-autonomous activity. To test this hypothesis, we used the scFv-Otx2 mouse model, which conditionally expresses a single-chain antibody (scFv) that blocks transfer by sequestering OTX2 in the CSF (Bernard et al., 2016). Cre-Tat intracerebroventricular injection leads to local scFv-Otx2 expression by ChP cells (GFP reporter; Fig. $4 A$ ) and secretion into the CSF, which can lead to $\sim 20 \%$ decrease in cortical OTX2 levels after 2 weeks (Bernard et al., 2016). Here, $35 \mathrm{~d}$ after Cre-Tat intracerebroventricular injection, this sequestering of Otx2 in CSF led to $\sim 10 \%$ decrease in OTX2 levels within V-SVZ (Fig. 4B,C), although it was not statistically significant given the large distribution of OTX2 mean intensity per cell (Fig. 4C). Subjecting these mice to the BrdU paradigm for OB integration (Fig. $4 D$ ) revealed a decrease in newborn neurons in the GCL of Cre-Tatinjected animals and no significant change in the GL (Fig. $4 E$ ). TUNEL assay revealed no change in neuroblast survival on arrival in the OB (Fig. 4F). Together, these results are similar to what was observed in Otx2-lox mice (Fig. $1 F, H)$ and suggest that the transfer of OTX2 from CSF to V-SVZ plays a significant role in regulating adult neurogenesis. The accumulation of OTX2 protein in supporting cells (Fig. 3) within the niche further suggests that OTX2 regulates V-SVZ neurogenesis through modification of the niche microenvironment.

\section{OTX2 regulates astrocyte factors}

As we observe OTX2 internalization all along the migrating route of neuroblasts (Fig. 3 ) and found decreased neuroblast migration (Fig. $1 \mathrm{~N}$ ) with no change in cell
A
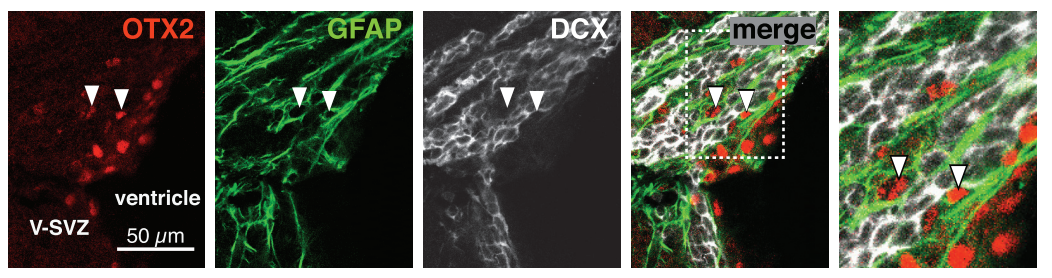

C
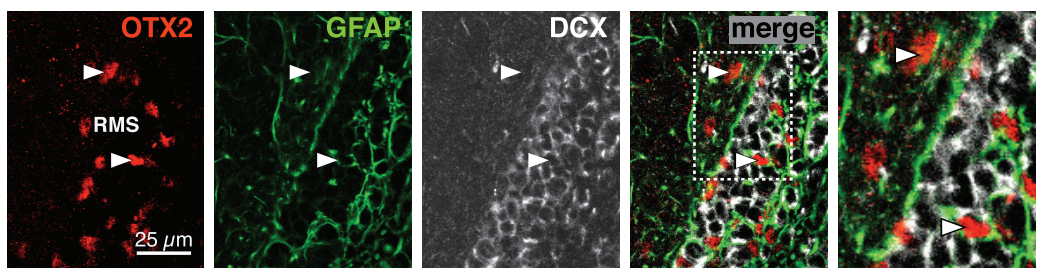

B
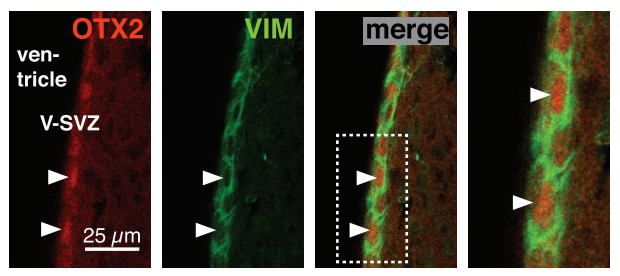

D

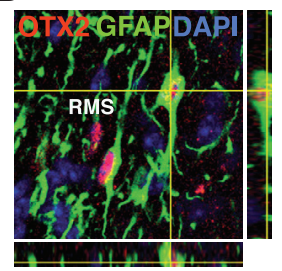

Figure 3. OTX2 transfers into supporting cells of neurogenic niches. $\boldsymbol{A}$, In dorsal V-SVZ, OTX2 staining is detected in some GFAP-labeled astrocytes but not in DCX-labeled neuroblasts. Arrowheads highlight astrocytes containing OTX2. B, Along the lateral ventricular wall, OTX2 staining is detected in vimentin (VIM)-labeled ependymal cells. $\boldsymbol{C}$, In the RMS, OTX2 staining is detected in GFAP-labeled astrocytes but not in DCX-labeled neuroblasts. Arrowheads highlight astrocytes containing OTX2. D, Orthogonal projection shows OTX2 in the nucleus of astrocytes within the RMS. 
A

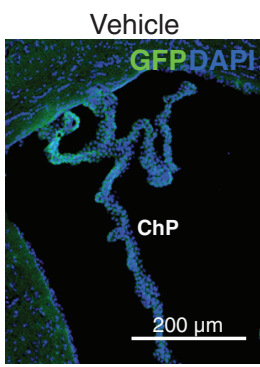

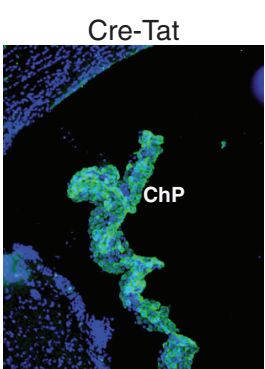

B
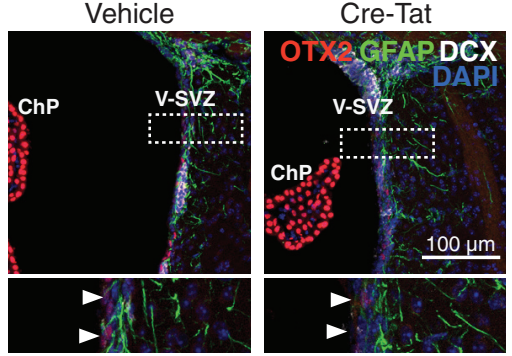

C

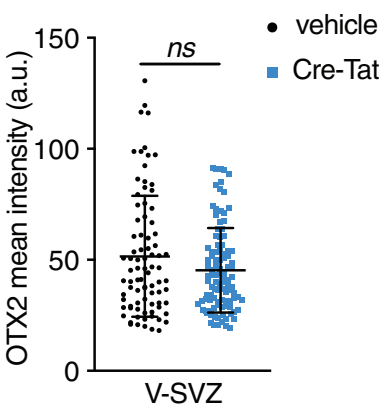

D

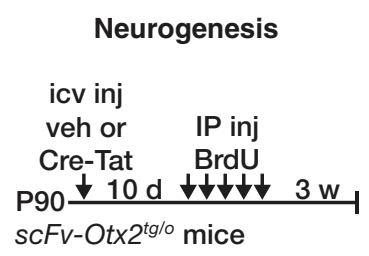

E

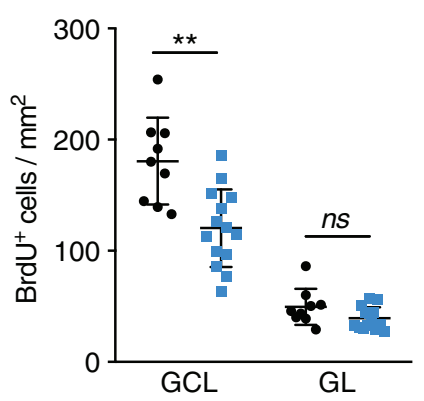

F

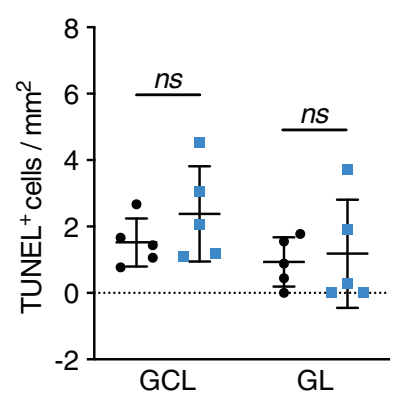

Figure 4. Non-cell-autonomous OTX2 is sufficient to regulate adult neurogenesis. $\boldsymbol{A}$, Efficient recombination in choroid plexus of scFv-Otx2 mice after intracerebroventricular injection of Cre-Tat assessed by GFP reporter. $\boldsymbol{B}$, Change in OTX2 staining within V-SVZ $35 \mathrm{~d}$ after Cre-Tat intracerebroventricular injection. C, Quantification of OTX2 mean intensity per cell in V-SVZ (ns $=p>0.05$, Mann-Whitney test). $\boldsymbol{D}$, Schematic of adult (3 months old) neurogenesis study paradigm with scFv-Otx2 mice. $\boldsymbol{E}$, Quantification of BrdU-positive cells in olfactory bulb GCL and GL ( $\mathrm{ns}=p>0.05, * * p<0.01, t$ test, 3 experiments). $\boldsymbol{F}$, TUNEL analysis of GCL and GL to assess cell death in olfactory bulb of scFv-Otx2 mice (ns $=p>0.05$, Mann-Whitney test, 2 experiments). IP, Intraperitoneal; icv, intracerebroventricular; inj, injection; veh, vehicle. Error bars represent the SD.

proliferation (Fig. $1 K$ ), we focused on a possible role for astrocytes found in both V-SVZ and RMS. Several receptors and factors are expressed in V-SVZ and RMS astrocytes that could potentially alter microenvironment composition and signaling. These include ECM proteins such as thrombospondin (Thbs1 and Thbs4) and tenascin-C (Tnc; Jankovski and Sotelo, 1996; Girard et al., 2014) and signaling molecules such as Robo and Eph receptors along with Ephrin ligands (Kaneko et al., 2010; Ming and Song, 2011; Falcão et al., 2012; Gengatharan et al., 2016; Todd et al., 2017), which may be under transcriptional control of OTX2 (Gherzi et al., 1997; Boncinelli and Morgan, 2001; Hoch et al., 2015; Peña et al.,
2017). Expression analysis on V-SVZ microdissection lysates from the scFv-Otx2 non-cell-autonomous OTX2 knock-down model showed that EphrinA1, Thbs1, Thbs4, and Tnc were upregulated in V-SVZ (Fig. 5A). We also found that both Thbs 4 and Tnc were upregulated in V-SVZ of Cre-Tat-injected Otx2-lox mice [mean \pm SD: $304.6 \pm 114.5 \%$ of vehicle $(p<0.0001)$ and $201.1 \pm 94 \%$ of vehicle $(p=0.0037)$, respectively], reinforcing the similarity between both mouse models.

To confirm that supporting astrocytes are directly implicated in these changes in the expression of ECM and signaling factors, we turned to an in vitro astrocyte model involving aged astrocytes from newborn mouse cortex to
A

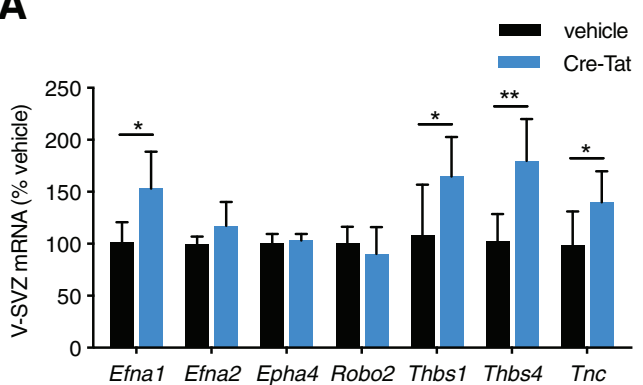

B

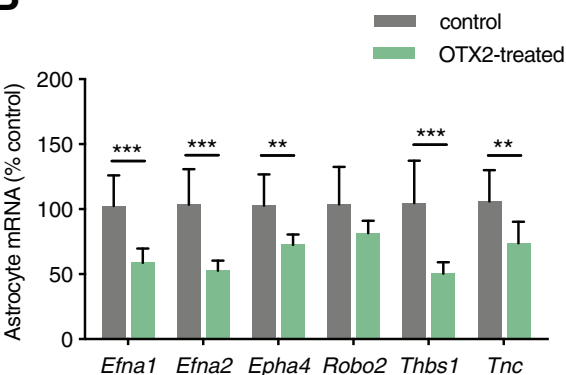

Figure 5. OTX2 regulates expression of ECM and signaling proteins. A, Quantitative PCR analysis of V-SVZ gene expression in scFv-Otx2 after intracerebroventricular injection of Cre-Tat or vehicle (ns $=p>0.05, * p<0.05, * * p<0.01$, Mann-Whitney test). $\boldsymbol{B}$, Quantitative PCR analysis of OTX2-treated astrocyte cultures (**p $<0.01$, ***p $<0.001$, Mann-Whitney test). Error bars represent the SD. 
provide mature astrocytes that do not contain NSCs typical of V-SVZ cultures. Indeed, cultured late juvenile and adult cortex astrocytes do not give rise to neurons, while cultured V-SVZ astrocytes retain this potential (Laywell et al., 2000). However, astrocyte cultures derived from early juvenile mouse cortex show low Thbs 4 expression (Benner et al., 2013), and cultured astrocytes typically show enriched expression of Thbs 1 and repression of Thbs4 compared with their in vivo counterparts (Cahoy et al., 2008; Eroglu, 2009). While astrocytes in our in vitro model do not perfectly represent isolated V-SVZ supporting cells, they are suitable for assessing non-cell-autonomous OTX2 activity on mature and differentiated astrocytes given the absence of confounding NSCs. Consistent with changes observed in vivo, treatment with OTX2 protein resulted in the downregulation of EphrinA1, Thbs1, and Tnc (Fig. 5B), with the exception of Thbs4, which is not expressed in our cultured astrocytes and is in keeping with previous studies. In this in vitro paradigm, OTX2 was also found to regulate EphrinA2 and Eph4 expression, which further highlights differences with adult V-SVZ astrocytes. Nonetheless, these results are in agreement with OTX2 transfer to niche astrocytes to regulate neurogenesis by controlling ECM composition and signaling.

\section{Discussion}

OTX2 homeoprotein was previously found to act noncell-autonomously in cortical interneurons (Sugiyama et al., 2008; Spatazza et al., 2013; Lee et al., 2017). The Otx2 locus is not active in the V-SVZ and RMS, but the protein can be immunoprecipitated, indicating that OTX2 homeoprotein transfers not only into several distant cortical regions but also into regions proximal to the lateral ventricles. In the cortex, OTX2 is specifically internalized by parvalbumin interneurons, which have a unique and stable ECM called perineuronal nets (PNNs) that often stain for the lectin Wisteria floribunda agglutinin (WFA; Sugiyama et al., 2008). The specificity of internalization arises from a glycosaminoglycan-binding motif in OTX2 protein (Beurdeley et al., 2012; Lee et al., 2017), and although specific target glycosaminoglycans of OTX2 have yet to be identified, OTX2 preferentially binds disulfated chondroitin sulfate proteoglycans (Beurdeley et al., 2012; Miyata et al., 2012; Bernard and Prochiantz, 2016). However, staining for WFA is not detected in the V-SVZ (data not shown), which is not surprising given that PNNs are synonymous with consolidation of ECM and a break on plasticity-events that would counter the function of the neurogenic niche. The hypothesis of ECM-OTX2 interaction accounting for the specific internalization remains the most plausible, given that homeoprotein cellsurface receptors have not been found (Bernard and Prochiantz, 2016). Thus, the specificity of OTX2 for astrocytes within the V-SVZ and RMS suggests that they are expressing glycosaminoglycans with high affinity for OTX2.

Through specific conditional recombination in the ChP of two mouse models, similar decreases in neurogenesis were observed. In Otx2-lox mice, recombination results in loss of local OTX2 expression in the ChP, while in scFvOtx2 mice it results in sequestering OTX2 in the CSF due to single-chain antibody secretion by the ChP (Bernard et al., 2016). The first model includes both potential cellautonomous effects of loss of OTX2 in the ChP as well as potential non-cell-autonomous effects through reduced levels of secreted OTX2. The second model comprises only non-cell-autonomous effects as only secreted OTX2 is affected. This is confirmed by the absence of autocrine effects (i.e., CSF OTX2 signaling on ChP) assessed by ChP mRNA levels of OTX2 transcription targets on scFv expression (Bernard et al., 2016). Since both models provide a similar reduction in the number of newborn neurons, it suggests that OTX2 non-cell-autonomous function is of primary importance for adult neurogenesis. Interestingly, we observe an effect despite a nonsignificant reduction in OTX2 accumulation in V-SVZ, which is likely due in part to the large distribution of mean OTX2 staining intensity. Small $(\sim 20 \%)$ decreases in OTX2 in adult mouse visual cortex induce cortical plasticity (Spatazza et al., 2013; Bernard et al., 2016), and OTX2 transcriptional targets can be activated at one concentration of OTX2 yet be suppressed at lower or higher concentrations (Apulei et al., 2018). Concentration-dependent targets of homeoprotein are shown to be dependent on chromatin state, whereby some targets are concentration insensitive while others are highly concentration sensitive (Hannon et al., 2017). Indeed, we find significant changes in some but not all putative targets of OTX2 in the V-SVZ despite the potentially subtle changes in OTX2 accumulation.

Previous studies on the role of factors secreted by the ChP mainly show effects on the proliferation of NSCs and transit-amplifying cells, as well as effects on maintaining NSC quiescence and the migration of neuroblasts (Bjornsson et al., 2015; Silva-Vargas et al., 2016). These factors were found to signal through quiescent and/or activated NSCs, such as for BMP5, IGF2, or NT-3 (Delgado et al., 2014; Ziegler et al., 2015; Silva-Vargas et al., 2016), or were found to signal directly through neuroblasts to regulate migration, such as for SLIT1/2 (Nguyen-Ba-Charvet et al., 2004). Interestingly, ROBO2 is expressed by supporting astrocytes within the V-SVZ and RMS and influences the migration of neuroblasts, which express SLIT1 (Kaneko et al., 2010). This suggests that SLIT1/2 from $\mathrm{ChP}$ can also be signaling through supporting astrocytes. We find the ChP knockdown of OTX2 reduces GCL newborn neurons without significantly modifying of cell proliferation or cell death but by modifying neuroblast migration into the RMS. However, one might expect reduced neuroblast migration to lead to a proportional reduction in dying neuroblasts. Yet cell death is difficult to study in this population given the spatiotemporal heterogeneity of their death and the high variability in their detection (Ryu et al., 2016). Thus, we cannot formally exclude that OTX2 is also affecting cell death. Nonetheless, OTX2 protein transfers in GFAP-positive cells in the V-SVZ, in the majority of GFAP-positive cells in the RMS, and in ependymal cells of the V-SVZ; this profile points to 
modification of the neurogenic niche instead of direct modification of progenitor activity.

Given the precedent of ECM and supporting astrocytes for regulating neuroblast migration (Gengatharan et al., 2016; Kaneko et al., 2017), we examined OTX2dependent expression of local ECM and signaling molecules expressed within V-SVZ and RMS. The RMS is composed of a compacted neuroblast network forming chains that migrate along blood vessels and are surrounded by astrocytic processes (Lois et al., 1996; Whitman et al., 2009). While it is clear that RMS-associated astrocytes interact with neuroblasts (Kaneko et al., 2010), the role of astrocytes for neuroblast migration is not well known. Studies show that neurogenesis is impacted by the composition of ECM in stem cell niches (Dityatev et al., 2010), which include astrocyte-derived TNC and THBS4 in V-SVZ and RMS (Jankovski and Sotelo, 1996; Benner et al., 2013; Girard et al., 2014), and OTX2 has been shown to regulate Tnc and Thbs4 transcription (Gherzi et al., 1997; Peña et al., 2017). While Tnc knock-out mice show no signs of altered adult neurogenesis (Kazanis et al., 2007), Thbs 4 knock-out adult mice show reduced newborn neurons in the OB, yet no defect of embryonic neurogenesis (Girard et al., 2014). Cellautonomous OTX2 expression was found to parallel Thbs4 expression in the retina (Housset et al., 2013), suggesting that OTX2 activity is context dependent, as previously suggested (Buecker et al., 2014). However, given the global dysregulation observed, we cannot come to a conclusion on the role of the different ECM molecules and interacting proteins. Nevertheless, changes in V-SVZ expression of Thc and Thbs4 on Otx2 knockdown are in keeping with a transcriptional role for non-cell-autonomous OTX2 in astrocytes.

As OTX2 was found in RMS astrocytes and signalization with neuroblasts is still an open question (Chaker et al., 2016), we tested whether genes coding for migration and signaling factors might be modified in our models. Robo2 is strongly expressed in RMS astrocytes and is required for proper RMS organization and guidance of neuroblasts (Kaneko et al., 2010), and it has been hypothesized that astrocyte-derived laminin helps to form RMS scaffold for neuroblast migration (Belvindrah et al., 2007). Also, EphA4 and EphrinA2 are expressed in RMS and V-SVZ, supporting astrocytes (along with other combinations of Ephs and Ephrins), and EPHA4 has been shown to be critical for maintaining a compact and organized RMS (Todd et al., 2017). While OTX2 has been shown to bind Robo2 promoter (Hoch et al., 2015) and to regulate Ephrin-A2 (Rhinn et al., 1999), we did not detect any changes in V-SVZ expression after OTX2 knockdown. However, OTX2 affected expression more widely in cultured mature astrocytes, including the expression of EphA4 and EphrinA2, suggesting that it has the potential to influence RMS organization and/or that its activity is differentially regulated among astrocyte subtypes. While these cultured astrocytes are not derived from V-SVZ, they reflect better the activity of OTX2 in supporting astrocytes given that V-SVZ cultures are dominated by GFAP-expressing NSCs. This in vitro model confirms that transfer of OTX2 into V-SVZ can regulate the expression of genes found in supporting astrocytes.

Our results add to the growing research on homeoprotein transduction (Di Nardo et al., 2018) and add to the complexity of ChP function, which is essential for brain homeostasis and neuroimmune regulation (Spector et al., 2015; Ghersi-Egea et al., 2018). OTX2 signaling through the CSF to cortical interneurons has been shown to regulate brain plasticity throughout the cortex and impact higher cognitive functions. We must now consider that ChP-derived OTX2 accumulates in diverse regions of the brain and that altering its concentration within CSF can influence a wide range of brain functions. Within the V-SVZ, OTX2 regulates ECM molecules and factors that likely impact neuroblast migration and ultimately newborn neuron numbers. These findings reinforce both $\mathrm{ChP}$ and V-SVZ non-neurogenic astrocytes as key niche compartments affecting adult neurogenesis.

\section{References}

Acampora D, Di Giovannantonio LG, Di Salvio M, Mancuso P, Simeone A (2009) Selective inactivation of Otx2 mRNA isoforms reveals isoform-specific requirement for visceral endoderm anteriorization and head morphogenesis and highlights cell diversity in the visceral endoderm. Mech Dev 126:882-897.

Acampora D, Omodei D, Petrosino G, Garofalo A, Savarese M, Nigro V, Di Giovannantonio LG, Mercadante V, Simeone A (2016) Loss of the Otx2-binding site in the Nanog promoter affects the integrity of embryonic stem cell subtypes and specification of inner cell massderived epiblast. Cell Rep 15:2651-2664.

Apulei J, Kim N, Testa D, Ribot J, Morizet D, Bernard C, Jourdren L, Blugeon C, Di Nardo AA, Prochiantz A (2018) Non-cell autonomous OTX2 homeoprotein regulates visual cortex plasticity through Gadd45b/g. Cereb Cortex. Advance online publication. Retrieved May 16, 2018. doi:10.1093/cercor/bhy108.

Belvindrah R, Hankel S, Walker J, Patton BL, Müller U (2007) Beta1 integrins control the formation of cell chains in the adult rostral migratory stream. J Neurosci 27:2704-2717.

Benner EJ, Luciano D, Jo R, Abdi K, Paez-Gonzalez P, Sheng H, Warner DS, Liu C, Eroglu C, Kuo CT (2013) Protective astrogenesis from the SVZ niche after injury is controlled by Notch modulator Thbs4. Nature 497:369-373.

Bernard C, Prochiantz A (2016) Otx2-PNN interaction to regulate cortical plasticity. Neural Plast 2016:7931693.

Bernard C, Vincent C, Testa D, Bertini E, Ribot J, Di Nardo AA, Volovitch M, Prochiantz A (2016) A mouse model for conditional secretion of specific single-chain antibodies provides genetic evidence for regulation of cortical plasticity by a non-cell autonomous homeoprotein transcription factor. PLoS Genet 12: e1006035.

Beurdeley M, Spatazza J, Lee HHC, Sugiyama S, Bernard C, Di Nardo AA, Hensch TK, Prochiantz A (2012) Otx2 binding to perineuronal nets persistently regulates plasticity in the mature visual cortex. J Neurosci 32:9429-9437.

Bjornsson CS, Apostolopoulou M, Tian Y, Temple S (2015) It takes a village: constructing the neurogenic niche. Dev Cell 32:435-446.

Boncinelli E, Morgan R (2001) Downstream of Otx2, or how to get a head. Trends Genet 17:633-636.

Buecker C, Srinivasan R, Wu Z, Calo E, Acampora D, Faial T, Simeone A, Tan M, Swigut T, Wysocka J (2014) Reorganization of enhancer patterns in transition from naive to primed pluripotency. Cell Stem Cell 14:838-853.

Cahoy JD, Emery B, Kaushal A, Foo LC, Zamanian JL, Christopherson KS, Xing Y, Lubischer JL, Krieg PA, Krupenko SA, Thompson WJ, Barres BA (2008) A transcriptome database for astrocytes, 
neurons, and oligodendrocytes: a new resource for understanding brain development and function. J Neurosci 28:264-278.

Chaker Z, Codega P, Doetsch F (2016) A mosaic world: puzzles revealed by adult neural stem cell heterogeneity. Wiley Interdiscip Rev Dev Biol 5:640-658.

Curto GG, Nieto-Estévez V, Hurtado-Chong A, Valero J, Gómez C, Alonso JR, Weruaga E, Vicario-Abejón C (2014) Pax6 is essential for the maintenance and multi-lineage differentiation of neural stem cells, and for neuronal incorporation into the adult olfactory bulb. Stem Cells Dev 23:2813-2830.

Delgado AC, Ferrón SR, Vicente D, Porlan E, Perez-Villalba A, Trujillo CM, D'Ocón P, Fariñas I (2014) Endothelial NT-3 delivered by vasculature and CSF promotes quiescence of subependymal neural stem cells through nitric oxide induction. Neuron 83:572-585.

Di Nardo AA, Fuchs J, Joshi RL, Moya KL, Prochiantz A (2018) The physiology of homeoprotein transduction. Physiol Rev 98:19431982.

Dityatev A, Seidenbecher Cl, Schachner M (2010) Compartmentalization from the outside: the extracellular matrix and functional microdomains in the brain. Trends Neurosci 33:503-512.

Eroglu C (2009) The role of astrocyte-secreted matricellular proteins in central nervous system development and function. J Cell Commun Signal 3:167-176.

Falcão AM, Marques F, Novais A, Sousa N, Palha JA, Sousa JC (2012) The path from the choroid plexus to the subventricular zone: go with the flow! Front Cell Neurosci 6:34.

Ferrón SR, Charalambous M, Radford E, McEwen K, Wildner H, Hind E, Morante-Redolat JM, Laborda J, Guillemot F, Bauer SR, Fariñas I, Ferguson-Smith AC (2011) Postnatal loss of Dlk1 imprinting in stem cells and niche astrocytes regulates neurogenesis. Nature 475:381-385.

Fiorelli R, Azim K, Fischer B, Raineteau O (2015) Adding a spatial dimension to postnatal ventricular-subventricular zone neurogenesis. Development 142:2109-2120.

Fossat N, Chatelain G, Brun G, Lamonerie T (2006) Temporal and spatial delineation of mouse Otx2 functions by conditional selfknockout. EMBO Rep 7:824-830.

Gengatharan A, Bammann RR, Saghatelyan A (2016) The role of astrocytes in the generation, migration, and integration of new neurons in the adult olfactory bulb. Front Neurosci 10:149.

Ghersi-Egea J-F, Strazielle N, Catala M, Silva-Vargas V, Doetsch F, Engelhardt B (2018) Molecular anatomy and functions of the choroidal blood-cerebrospinal fluid barrier in health and disease. Acta Neuropathol 135:337-361.

Gherzi R, Briata P, Boncinelli E, Ponassi M, Querzè G, Viti F, Corte G, Zardi $L$ (1997) The human homeodomain protein OTX2 binds to the human tenascin- $C$ promoter and trans-represses its activity in transfected cells. DNA Cell Biol 16:559-567.

Girard F, Eichenberger S, Celio MR (2014) Thrombospondin 4 deficiency in mouse impairs neuronal migration in the early postnatal and adult brain. Mol Cell Neurosci 61:176-186.

Hannon CE, Blythe SA, Wieschaus EF (2017) Concentration dependent chromatin states induced by the bicoid morphogen gradient. Elife 6:3165.

Hoch RV, Lindtner S, Price JD, Rubenstein JLR (2015) OTX2 transcription factor controls regional patterning within the medial ganglionic eminence and regional identity of the septum. Cell Rep 12:482-494.

Housset M, Samuel A, Ettaiche M, Bemelmans A, Béby F, Billon N, Lamonerie T (2013) Loss of otx2 in the adult retina disrupts retinal pigment epithelium function, causing photoreceptor degeneration. J Neurosci 33:9890-9904.

Jankovski A, Sotelo C (1996) Subventricular zone-olfactory bulb migratory pathway in the adult mouse: cellular composition and specificity as determined by heterochronic and heterotopic transplantation. J Comp Neurol 371:376-396.

Kaneko N, Marín O, Koike M, Hirota Y, Uchiyama Y, Wu JY, Lu Q, Tessier-Lavigne M, Alvarez-Buylla A, Okano H, Rubenstein JLR,
Sawamoto K (2010) New neurons clear the path of astrocytic processes for their rapid migration in the adult brain. Neuron 67:213-223.

Kaneko N, Sawada M, Sawamoto K (2017) Mechanisms of neuronal migration in the adult brain. J Neurochem 141:835-847.

Kazanis I, Belhadi A, Faissner A, Ffrench-Constant C (2007) The adult mouse subependymal zone regenerates efficiently in the absence of tenascin-C. J Neurosci 27:13991-13996.

Kokovay E, Wang Y, Kusek G, Wurster R, Lederman P, Lowry N, Shen Q, Temple S (2012) VCAM1 is essential to maintain the structure of the SVZ niche and acts as an environmental sensor to regulate SVZ lineage progression. Cell Stem Cell 11:220-230.

Laywell ED, Rakic P, Kukekov VG, Holland EC, Steindler DA (2000) Identification of a multipotent astrocytic stem cell in the immature and adult mouse brain. Proc Natl Acad Sci U S A 97:13883-13888.

Lee HHC, Bernard C, Ye Z, Acampora D, Simeone A, Prochiantz A, Di Nardo AA, Hensch TK (2017) Genetic Otx2 mis-localization delays critical period plasticity across brain regions. Mol Psychiatry 22:680-688.

Lehtinen MK, Bjornsson CS, Dymecki SM, Gilbertson RJ, Holtzman DM, Monuki ES (2013) The choroid plexus and cerebrospinal fluid: emerging roles in development, disease, and therapy. J Neurosci 33:17553-17559.

Lledo P-M, Valley M (2016) Adult olfactory bulb neurogenesis. Cold Spring Harb Perspect Biol 8:a018945.

Lois C, García-Verdugo JM, Alvarez-Buylla A (1996) Chain migration of neuronal precursors. Science 271:978-981.

Même W, Calvo C-F, Froger N, Ezan P, Amigou E, Koulakoff A, Giaume C (2006) Proinflammatory cytokines released from microglia inhibit gap junctions in astrocytes: potentiation by betaamyloid. FASEB J 20:494-496.

Ming G-L, Song H (2011) Adult neurogenesis in the mammalian brain: significant answers and significant questions. Neuron 70 : 687-702.

Mirzadeh Z, Merkle FT, Soriano-Navarro M, Garcia-Verdugo JM, Alvarez-Buylla A (2008) Neural stem cells confer unique pinwheel architecture to the ventricular surface in neurogenic regions of the adult brain. Cell Stem Cell 3:265-278.

Mirzadeh Z, Doetsch F, Sawamoto K, Wichterle H, Alvarez-Buylla A (2010) The subventricular zone en-face: wholemount staining and ependymal flow. J Vis Exp 6:1938.

Miyata S, Komatsu Y, Yoshimura Y, Taya C, Kitagawa H (2012) Persistent cortical plasticity by upregulation of chondroitin 6-sulfation. Nat Neurosci 15:414-422.

Nguyen-Ba-Charvet KT, Picard-Riera N, Tessier-Lavigne M, BaronVan Evercooren A, Sotelo C, Chédotal A (2004) Multiple roles for slits in the control of cell migration in the rostral migratory stream. J Neurosci 24:1497-1506.

Peña CJ, Kronman HG, Walker DM, Cates HM, Bagot RC, Purushothaman I, Issler O, Loh Y-H, Leong T, Kiraly DD, Goodman E, Neve RL, Shen L, Nestler EJ (2017) Early life stress confers lifelong stress susceptibility in mice via ventral tegmental area OTX2. Science 356:1185-1188.

Petrik D, Myoga MH, Grade S, Gerkau NJ, Pusch M, Rose CR, Grothe B, Götz M (2018) Epithelial sodium channel regulates adult neural stem cell proliferation in a flow-dependent manner. Cell Stem Cell 22:865-878.e8.

Prochiantz A, Di Nardo AA (2015) Homeoprotein signaling in the developing and adult nervous system. Neuron 85:911-925.

Rhinn M, Dierich A, Le Meur M, Ang S (1999) Cell autonomous and non-cell autonomous functions of Otx2 in patterning the rostral brain. Development 126:4295-4304.

Ryu JR, Hong CJ, Kim JY, Kim E-K, Sun W, Yu S-W (2016) Control of adult neurogenesis by programmed cell death in the mammalian brain. Mol Brain 9:43.

Sawamoto K, Wichterle H, Gonzalez-Perez O, Cholfin JA, Yamada M, Spassky N, Murcia NS, Garcia-Verdugo JM, Marin O, Rubenstein JLR, Tessier-Lavigne M, Okano H, Alvarez-Buylla A (2006) 
New neurons follow the flow of cerebrospinal fluid in the adult brain. Science 311:629-632.

Shen Q, Wang Y, Kokovay E, Lin G, Chuang S-M, Goderie SK, Roysam B, Temple S (2008) Adult SVZ stem cells lie in a vascular niche: a quantitative analysis of niche cell-cell interactions. Cell Stem Cell 3:289-300.

Silva-Vargas V, Crouch EE, Doetsch F (2013) Adult neural stem cells and their niche: a dynamic duo during homeostasis, regeneration, and aging. Curr Opin Neurobiol 23:935-942.

Silva-Vargas V, Maldonado-Soto AR, Mizrak D, Codega P, Doetsch F (2016) Age-dependent niche signals from the choroid plexus regulate adult neural stem cells. Cell Stem Cell 19:643-652.

Spatazza J, Lee HHC, Di Nardo AA, Tibaldi L, Joliot A, Hensch TK, Prochiantz A (2013) Choroid-plexus-derived Otx2 homeoprotein constrains adult cortical plasticity. Cell Rep 3:1815-1823.

Spector R, Keep RF, Robert Snodgrass S, Smith QR, Johanson CE (2015) A balanced view of choroid plexus structure and function: focus on adult humans. Exp Neurol 267:78-86.
Sugiyama S, Di Nardo AA, Aizawa S, Matsuo I, Volovitch M, Prochiantz A, Hensch TK (2008) Experience-dependent transfer of Otx2 homeoprotein into the visual cortex activates postnatal plasticity. Cell 134:508-520.

Todd KL, Baker KL, Eastman MB, Kolling FW, Trausch AG, Nelson CE, Conover JC (2017) EphA4 regulates neuroblast and astrocyte organization in a neurogenic niche. J Neurosci 37:33313341.

Whitman MC, Fan W, Rela L, Rodriguez-Gil DJ, Greer CA (2009) Blood vessels form a migratory scaffold in the rostral migratory stream. J Comp Neurol 516:94-104.

Yang S-H, Kalkan T, Morissroe C, Marks H, Stunnenberg H, Smith A, Sharrocks AD (2014) Otx2 and Oct4 drive early enhancer activation during embryonic stem cell transition from naive pluripotency. Cell Rep 7:1968-1981.

Ziegler AN, Levison SW, Wood TL (2015) Insulin and IGF receptor signalling in neural-stem-cell homeostasis. Nat Rev Endocrinol 11:161-170. 